\title{
Nachträge zur Bibliographie der Schriften von ALWIN DIEMER*
}

Phénoménologie de la Culture, in: Philosophy and Cultures. Proceedings of the 2nd Afro-Asian Philosophy Conference Nairobi Oct./Nov. 1981, ed. by H.O.Oruka/D.A. Masolo, Nairobi 1983, P. 30-43.

Wissenschaft (nach Material von Alwin Diemer $\dagger$, bearbeitet von Helmut Seiffert), in: Seiffert, H./ G. Radnitzky (Hg.), Handlexikon zur Wissenschaftstheorie, München 1989, S. 391-399.

*Vgl. diese Zeitschrift Band XVIII (1987), S. 355-359.

\section{EINGEGANGENE BÜCHER}

Agazzi, Evandro (Hg.): Probability in the Sciences (Synthese Library 201), Dordrecht, Boston, London 1988 (Kluwer), X + 269 Index (\$59).

Amrine, Frederick (Hg.): Literature and Science as Modes of Expression (Boston Studies in the Philosophy of Science 115), Dordrecht, Boston, London 1989 (Kluwer), XXV + 195 Index (\$67).

Audretsch, Jürgen/Klaus Mainzer (Hg.): Vom Anfang der Welt. Wissenschaft, Philosophie, Religion, Mythos, München 1989 (C. H. Beck), 228 Index.

Balzer, Wolfgang/Bert Hamminga (Hg.): Phylosophy of Economics (Erkenntnis 30, Nos. 1-2), Dordrecht, Boston, London 1989 (Kluwer), 270 (\$69).

Barut, A. O./A. van der Merwe ( $\mathrm{Hg}$.): Selected Scientific Papers of Alfred Landé (Fundamental Theories of Physics), Dordrecht, Boston, Lancaster Tokyo 1988 (D. Reidel), XII + 557 Index (\$99).

Borzeszkowski, H.-H. von/H.-J.Treder: The Meaning of Quantum Gravity (Fundamental Theories of Physics), Dordrecht, Boston, Lancaster Tokyo 1988 (D. Reidel), VI + 132 Index (\$48).

Butts, Robert E./James Robert Brown ( $\mathrm{Hg}$.): Constructivism and Science. Essays in recent German philosophy (The University of Western Ontario Series in Philosophy of Science 44), Dordrecht, Boston, London 1989 (Kluwer), XXV + 287 Index (\$96).

Byrne, Edmund F.JJoseph C. Pitt (Hg.): Technological Transformation. Contextual and Conceptual Implications (Official Publication of the Society for Philosophy and Technology 5), Dordrecht, Boston, London 1989 (Kluwer), XI + 314 Index (\$89).

Cartwright, Nancy: Natur's Capacities and their Measurement, Oxford 1989 (Clarendon Press), 268 Index.

Charpa, Ulrich (Hg.): Matthias Jakob Schleiden. Wissenschaftsphilosophische Schriften mit kommentierten Texten von Jakob Friedrich Fries, Christian G. Nees von Esenbeck und Gerd Buchdahl (Geschichte der Wissenschaftsphilosophie), Köln 1989 (J.Dinter), 418 Index (DM 120).

Chisholm, Roderick M./Johann C. Marek (Hg.): Franz Brentano, Über Ernst Machs ,Erkenntnis und Irrtum' (Studien zur österreichischen Philosophie VI), Amsterdam 1988 (Rodopi), 244 Index (Hfl 75).

Coreth, Emerich/Walter M.Neidl/Georg Pfligersdorffer $\left(\mathrm{Hg}_{\mathrm{s}}\right)$ : Christliche Philosophie im katholischen Denken des 19. und 20. Jahrhunderts, Bd. 2, Rückgriff auf das scholastische Erbe, Graz, Wien, Köln 1988 (Styria), 870 Index (DM 140).

D'Agostino, Fred/I. C. Jarvie (Hg.): Freedom and Rationality. Essays in Honor of John Watkins. From his Colleagues and Friends (Boston Studies in the Philosophy of Science 117), Dordrecht, Boston, London 1989 (Kluwer), XI + 388 Index (\$99). 